\title{
A unified approach to nonlocal impulsive differential equations with the measure of noncompactness
}

Shaochun $\mathrm{Ji}^{1,2^{*}}$ and Gang Li ${ }^{1}$

\section{"Correspondence:}

jiscmath@yahoo.com.cn

${ }^{1}$ School of Mathematical Science

Yangzhou University, Yangzhou,

225002, P.R. China

${ }^{2}$ Current address: Faculty of

Mathematics and Physics, Huaiyin Institute of Technology, Huaian,

223003, P.R. China

\begin{abstract}
This paper is concerned with the existence of mild solutions to impulsive differential equations with nonlocal conditions. We firstly establish a property of the measure of noncompactness in the space of piecewise continuous functions. Then, by applying this property and Darbo-Sadovskii's fixed point theorem, we get the existence results of impulsive differential equations in a unified way under compactness conditions, Lipschitz conditions and mixed-type conditions, respectively.
\end{abstract}

MSC: 34K30; 34G20

Keywords: impulsive conditions; nonlocal conditions; Hausdorff measure of noncompactness; fixed point theorem

\section{Introduction}

In this paper, we discuss the existence of mild solutions for the following impulsive differential equation with nonlocal conditions:

$$
\begin{aligned}
& u^{\prime}(t)=A u(t)+f(t, u(t)), \quad t \in J=[0, b], t \neq t_{i}, \\
& u(0)=g(u), \\
& \Delta u\left(t_{i}\right)=I_{i}\left(u\left(t_{i}\right)\right), \quad i=1, \ldots, s,
\end{aligned}
$$

where $A: D(A) \subseteq X \rightarrow X$ is the infinitesimal generator of a strongly continuous semigroup $T(t), t \geq 0$ in a Banach space $X, 0=t_{0}<t_{1}<t_{2}<\cdots<t_{s}<t_{s+1}=b, \Delta u\left(t_{i}\right)=u\left(t_{i}^{+}\right)-u\left(t_{i}^{-}\right)$, $u\left(t_{i}^{-}\right), u\left(t_{i}^{+}\right)$denote the left and right limit of $u$ at $t_{i}$, respectively. $f, g, I_{i}$ are appropriate functions to be specified later.

Impulsive differential equations are recognized as excellent models to study the evolution processes that are subject to sudden changes in their states; see the monographs of Lakshmikantham et al. [1], Benchohra et al. [2]. In recent years impulsive differential equations in Banach spaces have been investigated by many authors; see [3-8] and references therein. Liu [9] discussed the existence and uniqueness of mild solutions for a semilinear impulsive Cauchy problem with Lipschitz impulsive functions. Non-Lipschitzian impulsive equations are considered by Nieto et al. [10]. Cardinali and Rubbioni [11] proved the existence of mild solutions for the impulsive Cauchy problem controlled by a semilinear evolution differential inclusion. In [12], Abada et al. studied the existence of integral solutions for some nondensely defined impulsive semilinear functional differential inclusions.

\section{焦 Springer}

(c) $2012 \mathrm{Ji}$ and Li; licensee Springer. This is an Open Access article distributed under the terms of the Creative Commons Attribution License (http://creativecommons.org/licenses/by/2.0), which permits unrestricted use, distribution, and reproduction in any medium, provided the original work is properly cited. 
On the other hand, the study of abstract nonlocal initial value problems was initiated by Byszewski, and the importance of the problem consists in the fact that it is more general and has better effect than the classical initial conditions $u(0)=u_{0}$ alone. Therefore, it has been studied extensively under various conditions. Here we mention some results. Byszewski and Lakshmikantham [13], Byszewski [14] obtained the existence and uniqueness of mild solutions and classical solutions in the case that Lipschitz-type conditions are satisfied. In [15], Fu and Ezzinbi studied neutral functional-differential equations with nonlocal conditions. Aizicovici $[16]$, Xue $[17,18]$ discussed the case when $A$ generates a nonlinear contraction semigroup on $X$ and obtained the existence of integral solutions for nonlinear differential equations. In particular, the measure of noncompactness has been used as an important tool to deal with some similar functional differential and integral equations; see [18-22].

From the viewpoint of theory and practice, it is natural for mathematics to combine impulsive conditions and nonlocal conditions. Recently, the nonlocal impulsive differential problem of type (1.1) has been discussed in the papers of Liang et al. [23] and Fan et al. $[24,25]$, where a semigroup $T(t)$ is supposed to be compact, and $g$ is Lipschitz continuous, compact, and strongly continuous, respectively. Very recently, Zhu et al. [26] obtain the existence results when a nonlocal item $g$ is Lipschitz continuous by using the Hausdorff measure of noncompactness and operator transformation. Compared with the results in [23-25], in this paper we do not require the compactness of the semigroup $T(t)$ and Lipschitz continuity of $f$. More important, by using the property of the measure of noncompactness in $P C([0, b] ; X)$ given by us (see Lemma 2.7$)$, the impulsive conditions and nonlocal conditions can be considered in a unified way under various conditions, including compactness conditions, Lipschitz conditions and mixed-type conditions. Hence, our results generalize and partially improve the results in $[23,25,26]$.

This paper is organized as follows. In Section 2, we present some concepts and facts about the strongly continuous semigroup and the measure of noncompactness. In Section 3, we give four existence theorems of the problem (1.1) by using a condensing operator and the measure of noncompactness. At last, an example of an impulsive partial differential system is given in Section 4.

\section{Preliminaries}

Let $(X,\|\cdot\|)$ be a real Banach space. We denote by $C([0, b] ; X)$ the space of $X$-valued continuous functions on $[0, b]$ with the norm $\|x\|=\sup \{\|x(t)\|, t \in[0, b]\}$ and by $L^{1}([0, b] ; X)$ the space of $X$-valued Bochner integrable functions on $[0, b]$ with the norm $\|f\|_{L^{1}}=$ $\int_{0}^{b}\|f(t)\| \mathrm{d} t$.

The semigroup $T(t)$ is said to be equicontinuous if $\{T(t) x: x \in B\}$ is equicontinuous at $t>0$ for any bounded subset $B \subset X(c f$. [27]). Obviously, if $T(t)$ is a compact semigroup, it must be equicontinuous. And the converse of the relation usually is not correct. Throughout this paper, we suppose that

(HA) The semigroup $\{T(t): t \geq 0\}$ generated by $A$ is equicontinuous. Moreover, there exists a positive number $M$ such that $M=\sup _{0 \leq t \leq b}\|T(t)\|$.

For the sake of simplicity, we put $J=[0, b] ; J_{0}=\left[0, t_{1}\right] ; J_{i}=\left(t_{i}, t_{i+1}\right], i=1, \ldots, s$. In order to define a mild solution of the problem $(1.1)$, we introduce the set $P C([0, b] ; X)=\{u:[0, b] \rightarrow$ $X: u$ is continuous at $t \neq t_{i}$ and left continuous at $t=t_{i}$, and the right limit $u\left(t_{i}^{+}\right)$exists, $i=1, \ldots, s\}$. It is easy to verify that $P C([0, b] ; X)$ is a Banach space with the norm $\|u\|_{P C}=$ $\sup \{\|u(t)\|, t \in[0, b]\}$. 
Definition 2.1 A function $u \in P C([0, b] ; X)$ is a mild solution of the problem (1.1) if

$$
u(t)=T(t) g(u)+\int_{0}^{t} T(t-s) f(s, u(s)) \mathrm{d} s+\sum_{0<t_{i}<t} T\left(t-t_{i}\right) I_{i}\left(u\left(t_{i}\right)\right)
$$

for all $t \in[0, b]$.

Now, we introduce the Hausdorff measure of noncompactness (in short MNC) $\beta(\cdot)$ defined by

$$
\beta(B)=\inf \{\varepsilon>0 ; B \text { has a finite } \varepsilon \text {-net in } X\},
$$

for each bounded subset $B$ in a Banach space $X$. We recall the following properties of the Hausdorff measure of noncompactness $\beta$.

Lemma 2.2 ([28]) Let $X$ be a real Banach space and $B, C \subseteq X$ be bounded. Then the following properties are satisfied:

(1) $B$ is relatively compact if and only if $\beta(B)=0$;

(2) $\beta(B)=\beta(\bar{B})=\beta(\operatorname{conv} B)$, where $\bar{B}$ and conv $B$ mean the closure and convex hull of $B$, respectively;

(3) $\beta(B) \leq \beta(C)$ when $B \subseteq C$;

(4) $\beta(B+C) \leq \beta(B)+\beta(C)$, where $B+C=\{x+y: x \in B, y \in C\}$;

(5) $\beta(B \cup C) \leq \max \{\beta(B), \beta(C)\}$;

(6) $\beta(\lambda B) \leq|\lambda| \beta(B)$ for any $\lambda \in R$;

(7) If the map $Q: D(Q) \subseteq X \rightarrow Z$ is Lipschitz continuous with a constant $k$, then $\beta_{Z}(Q B) \leq k \beta(B)$ for any bounded subset $B \subseteq D(Q)$, where $Z$ is a Banach space.

The map $Q: D \subseteq X \rightarrow X$ is said to be $\beta$-condensing if $Q$ is continuous and bounded, and for any nonprecompact bounded subset $B \subset D$, we have $\beta(Q B)<\beta(B)$, where $X$ is a Banach space.

Lemma 2.3 (See [28], Darbo-Sadovskii) If $D \subset X$ is bounded, closed, and convex, the continuous map $Q: D \rightarrow D$ is $\beta$-condensing, then $Q$ has at least one fixed point in $D$.

In order to remove the strong restriction on the coefficient in Darbo-Sadovskii's fixed point theorem, Sun and Zhang [29] generalized the definition of a $\beta$-condensing operator. At first, we give some notation. Let $D \subset X$ be closed and convex, the map $Q: D \rightarrow D$ and $x_{0} \in D$. For every $B \subset D$, set

$$
Q^{\left(1, x_{0}\right)}(B)=Q(B), \quad Q^{\left(n, x_{0}\right)}(B)=Q\left(\overline{\operatorname{conv}}\left\{Q^{\left(n-1, x_{0}\right)} B, x_{0}\right\}\right),
$$

where $\overline{\text { conv }}$ means the closure of convex hull, $n=2,3, \ldots$.

Definition 2.4 Let $D \subset X$ be closed and convex. The map $Q: D \rightarrow D$ is said to be $\beta$-convex-power condensing if $Q$ is continuous, bounded and there exist $x_{0} \in D, n_{0} \in N$ such that for every nonprecompact bounded subset $B \subset D$, we have

$$
\beta\left(Q^{\left(n_{0}, x_{0}\right)}(B)\right)<\beta(B)
$$


Obviously, if $n_{0}=1$, then a $\beta$-convex-power condensing operator is $\beta$-condensing. Thus, the convex-power condensing operator is a generalization of the condensing operator. Now, we give the fixed point theorem about the convex-power condensing operator.

Lemma 2.5 ([29]) If $D \subset X$ is bounded, closed, and convex, the continuous map $Q: D \rightarrow D$ is $\beta$-convex-power condensing, then $Q$ has at least one fixed point in $D$.

Now, we give an important property of the Hausdorff $\mathrm{MNC}$ in $P C([0, b] ; X)$, which is an extension to the property of $\mathrm{MNC}$ in $C([0, b] ; X)$ and makes us deal with the impulsive differential equations from a unified perspective.

Lemma 2.6 ([28]) If $W \subseteq C([0, b] ; X)$ is bounded, then $\beta(W(t)) \leq \beta(W)$ for all $t \in[0, b]$, where $W(t)=\{u(t) ; u \in W\} \subseteq X$. Furthermore, if $W$ is equicontinuous on $[0, b]$, then $\beta(W(t))$ is continuous on $[0, b]$ and $\beta(W)=\sup \{\beta(W(t)), t \in[0, b]\}$.

By applying Lemma 2.6 , we shall extend the result to the space $P C([0, b] ; X)$.

Lemma 2.7 If $W \subseteq P C([0, b]$; $X)$ is bounded, then $\beta(W(t)) \leq \beta(W)$ for all $t \in[0, b]$, where $W(t)=\{u(t) ; u \in W\} \subseteq X$. Furthermore, suppose the following conditions are satisfied:

(1) $W$ is equicontinuous on $J_{0}=\left[0, t_{1}\right]$ and each $J_{i}=\left(t_{i}, t_{i+1}\right], i=1, \ldots, s$;

(2) $W$ is equicontinuous at $t=t_{i}^{+}, i=1, \ldots, s$.

Then $\sup _{t \in[0, b]} \beta(W(t))=\beta(W)$.

Proof For arbitrary $\varepsilon>0$, there exists $W_{i} \subseteq P C([0, b] ; X), 1 \leq i \leq n$, such that $W=\bigcup_{i=1}^{n} W_{i}$ and

$$
\operatorname{diam}\left(W_{i}\right) \leq 2 \beta(W)+2 \varepsilon, \quad i=1,2, \ldots, n,
$$

where $\operatorname{diam}(\cdot)$ denotes the diameter of a bounded set. Now, we have $W(t)=\bigcup_{i=1}^{n} W_{i}(t)$ for each $t \in[a, b]$, and

$$
\|x(t)-y(t)\| \leq\|x-y\| \leq \operatorname{diam}\left(W_{i}\right)
$$

for $x, y \in W_{i}$. From the above two inequalities, it follows that

$$
2 \beta(W(t)) \leq \operatorname{diam}\left(W_{i}(t)\right) \leq \operatorname{diam}\left(W_{i}\right) \leq 2 \beta(W)+2 \varepsilon .
$$

By the arbitrariness of $\varepsilon$, we get that $\beta(W(t)) \leq \beta(W)$ for every $t \in[0, b]$. Therefore, we have $\sup _{t \in[0, b]} \beta(W(t)) \leq \beta(W)$.

Next, if the conditions (1) and (2) are satisfied, it remains to prove that $\beta(W) \leq$ $\sup _{t \in[0, b]} \beta(W(t))$. We denote $\left.W\right|_{\bar{J}_{i}}$ by the restriction of $W$ on $\bar{J}_{i}=\left[t_{i}, t_{i+1}\right], i=0,1, \ldots, s$. That is, for $\left.x \in W\right|_{\bar{J}_{i}}$, define that

$$
x(t)= \begin{cases}x(t), & t_{i}<t \leq t_{i+1}, \\ x\left(t_{i}^{+}\right), & t=t_{i},\end{cases}
$$


and obviously $\left.W\right|_{\overline{J_{i}}}$ is equicontinuous on $\overline{J_{i}}$ due to the conditions (1) and (2). Then from Lemma 2.6, we have that

$$
\beta\left(\left.W\right|_{\bar{J}_{i}}\right)=\sup _{t \in \bar{J}_{i}} \beta\left(\left.W\right|_{\bar{J}_{i}}(t)\right) .
$$

Moreover, we define the map

$$
\Lambda: P C([0, b] ; X) \rightarrow C\left(\left[0, t_{1}\right] ; X\right) \times C\left(\left[t_{1}, t_{2}\right] ; X\right) \times \cdots \times C\left(\left[t_{s}, b\right] ; X\right)
$$

by $x \rightarrow\left(x_{0}, x_{1}, \ldots, x_{s}\right)$, where $x \in P C([0, b] ; X), x_{i}=\left.x\right|_{\overline{J_{i}}},\left\|\left(x_{0}, x_{1}, \ldots, x_{s}\right)\right\|=\max _{0 \leq i \leq s}\left\|x_{i}\right\|$. As $\Lambda$ is an isometric mapping, noticing the equicontinuity of $\left.W\right|_{\overline{J_{i}}}$ on $\overline{J_{i}}$, we have that

$$
\beta(W)=\beta\left(\left.W\right|_{\overline{J_{0}}} \times\left. W\right|_{\bar{J}_{1}} \times \cdots \times\left. W\right|_{\bar{J}_{s}}\right) \leq \max _{i} \beta\left(\left.W\right|_{\overline{J_{i}}}\right)=\max _{i} \sup _{t \in \overline{J_{i}}} \beta\left(\left.W\right|_{\bar{J}_{i}}(t)\right) .
$$

And from the fact that $\sup _{t \in \overline{\bar{J}_{i}}} \beta\left(\left.W\right|_{\overline{J_{i}}}(t)\right) \leq \sup _{t \in[0, b]} \beta(W(t))$, for each $i=0, \ldots, s$, we get that $\beta(W) \leq \sup _{t \in[0, b]} \beta(W(t))$. This completes the proof.

Lemma 2.8 ([28]) If $W \subset C([0, b] ; X)$ is bounded and equicontinuous, then $\beta(W(t))$ is continuous and

$$
\beta\left(\int_{0}^{t} W(s) \mathrm{d} s\right) \leq \int_{0}^{t} \beta(W(s)) \mathrm{d} s,
$$

for all $t \in[0, b]$, where $\int_{0}^{t} W(s) \mathrm{d} s=\left\{\int_{0}^{t} x(s) \mathrm{d} s: x \in W\right\}$.

Lemma 2.9 If the hypothesis (HA) is satisfied, i.e., $\{T(t): t \geq 0\}$ is equicontinuous, and $\eta \in L^{1}\left([0, b] ; R^{+}\right)$, then the set $\left\{\int_{0}^{t} T(t-s) u(s) \mathrm{d} s:\|u(s)\| \leq \eta(s)\right.$ for a.e. $\left.s \in[0, b]\right\}$ is equicontinuous for $t \in[0, b]$.

Proof We let $0 \leq t<t+h \leq b$ and have that

$$
\begin{aligned}
& \left\|\int_{0}^{t+h} T(t+h-s) u(s) \mathrm{d} s-\int_{0}^{t} T(t-s) u(s) \mathrm{d} s\right\| \\
& \leq\left\|\int_{0}^{t} T(t+h-s) u(s) \mathrm{d} s-\int_{0}^{t} T(t-s) u(s) \mathrm{d} s\right\| \\
& \quad+\int_{t}^{t+h}\|T(t+h-s) u(s)\| \mathrm{d} s .
\end{aligned}
$$

If $t=0$, then the right-hand side of (2.1) can be made small when $h$ is small independent of $u$. If $t>0$, then we can find a small $\varepsilon>0$ with $t-\varepsilon>0$. Then it follows from (2.1) that

$$
\begin{aligned}
& \left\|\int_{0}^{t} T(t+h-s) u(s) \mathrm{d} s-\int_{0}^{t} T(t-s) u(s) \mathrm{d} s\right\| \\
& \quad \leq\left\|T(h+\varepsilon) \int_{0}^{t-\varepsilon} T(t-\varepsilon-s) u(s) \mathrm{d} s-T(\varepsilon) \int_{0}^{t-\varepsilon} T(t-\varepsilon-s) u(s) \mathrm{d} s\right\| \\
& \quad+\left\|\int_{t-\varepsilon}^{t} T(t+h-s) u(s) \mathrm{d} s\right\|+\left\|\int_{t-\varepsilon}^{t} T(t-s) u(s) \mathrm{d} s\right\| .
\end{aligned}
$$


Here, as $T(t)$ is equicontinuous for $t>0$, thus

$$
\left\|[T(h+\varepsilon)-T(\varepsilon)] \int_{0}^{t-\varepsilon} T(t-\varepsilon-s) u(s) \mathrm{d} s\right\| \rightarrow 0, \quad \text { as } h \rightarrow 0,
$$

uniformly for $u$.

Then from (2.1), (2.2) and the absolute continuity of integrals, we get that $\left\{\int_{0}^{t} T(t-\right.$ $s) u(s) \mathrm{d} s,\|u(s)\| \leq \eta(s)$ for a.e. $s \in[0, b]\}$ is equicontinuous for $t \in[0, b]$.

\section{Main results}

In this section we give the existence results for the problem (1.1) under different conditions on $g$ and $I_{i}$ when the semigroup is not compact and $f$ is not compact or Lipschitz continuous, by using Lemma 2.7 and the generalized $\beta$-condensing operator. More precisely, Theorem 3.1 is concerned with the case that compactness conditions are satisfied. Theorem 3.4 deals with the case that Lipschitz conditions are satisfied. And mixed-type conditions are considered in Theorem 3.5 and Theorem 3.6.

Let $r$ be a finite positive constant, and set $B_{r}=\{x \in X:\|x\| \leq r\}, W_{r}=\{u \in P C([0, b] ; X)$ : $\left.u(t) \in B_{r}, t \in[0, b]\right\}$. We define the solution map $G: P C([0, b] ; X) \rightarrow P C([0, b] ; X)$ by

$$
(G u)(t)=T(t) g(u)+\int_{0}^{t} T(t-s) f(s, u(s)) \mathrm{d} s+\sum_{0<t_{i}<t} T\left(t-t_{i}\right) I_{i}\left(u\left(t_{i}\right)\right)
$$

with

$$
\begin{aligned}
& \left(G_{1} u\right)(t)=T(t) g(u) \\
& \left(G_{2} u\right)(t)=\int_{0}^{t} T(t-s) f(s, u(s)) \mathrm{d} s \\
& \left(G_{3} u\right)(t)=\sum_{0<t_{i}<t} T\left(t-t_{i}\right) I_{i}\left(u\left(t_{i}\right)\right),
\end{aligned}
$$

for all $t \in[0, b]$. It is easy to see that $u$ is the mild solution of the problem (1.1) if and only if $u$ is a fixed point of the map $G$.

We list the following hypotheses:

(Hf) $f:[0, b] \times X \rightarrow X$ satisfies the following conditions:

(i) $f(t, \cdot): X \rightarrow X$ is continuous for a.e. $t \in[0, b]$ and $f(\cdot, x):[0, b] \rightarrow X$ is measurable for all $x \in X$. Moreover, for any $r>0$, there exists a function $\rho_{r} \in L^{1}([0, b], R)$ such that

$$
\|f(t, x)\| \leq \rho_{r}(t)
$$

for a.e. $t \in[0, b]$ and $x \in B_{r}$.

(ii) there exists a constant $L>0$ such that for any bounded set $D \subset X$,

$$
\beta(f(t, D)) \leq L \beta(D)
$$

for a.e. $t \in[0, b]$.

(Hg1) $g: P C([0, b] ; X) \rightarrow X$ is continuous and compact.

(HI1) $I_{i}: X \rightarrow X$ is continuous and compact for $i=1, \ldots, s$. 
Theorem 3.1 Assume that the hypotheses (HA), (Hf), (Hg1), (HI1) are satisfied, then the nonlocal impulsive problem (1.1) has at least one mild solution on $[0, b]$ provided that there exists a constant $r>0$ such that

$$
M\left[\sup _{u \in W_{r}}\|g(u)\|+\left\|\rho_{r}\right\|_{L^{1}}+\sup _{u \in W_{r}} \sum_{i=1}^{s}\left\|I_{i}\left(u\left(t_{i}\right)\right)\right\|\right] \leq r .
$$

Proof We will prove that the solution map $G$ has a fixed point by using the fixed point theorem about the $\beta$-convex-power condensing operator.

Firstly, we prove that the map $G$ is continuous on $P C([0, b] ; X)$. For this purpose, let $\left\{u_{n}\right\}_{n=1}^{\infty}$ be a sequence in $P C([0, b] ; X)$ with $\lim _{n \rightarrow \infty} u_{n}=u$ in $P C([0, b] ; X)$. By the continuity of $f$ with respect to the second argument, we deduce that for each $s \in[0, b], f\left(s, u_{n}(s)\right)$ converges to $f(s, u(s))$ in $X$. And we have

$$
\begin{aligned}
\left\|G u_{n}-G u\right\|_{P C} \leq & M\left[\left\|g\left(u_{n}\right)-g(u)\right\|+\sum_{i=1}^{s}\left\|I_{i}\left(u_{n}\left(t_{i}\right)\right)-I_{i}\left(u\left(t_{i}\right)\right)\right\|\right] \\
& +M \int_{0}^{b}\left\|f\left(s, u_{n}(s)\right)-f(s, u(s))\right\| \mathrm{d} s .
\end{aligned}
$$

Then by the continuity of $g, I_{i}$ and using the dominated convergence theorem, we get $\lim _{n \rightarrow \infty} G u_{n}=G u$ in $P C([0, b] ; X)$.

Secondly, we claim that $G W_{r} \subseteq W_{r}$. In fact, for any $u \in W_{r} \subset P C([0, b] ; X)$, from (3.1) and (3.3), we have

$$
\begin{aligned}
\|(G u)(t)\| & \leq\|T(t) g(u)\|+\left\|\int_{0}^{t} T(t-s) f(s, u(s)) \mathrm{d} s\right\|+\sum_{0<t_{i}<t}\left\|T\left(t-t_{i}\right) I_{i}\left(u\left(t_{i}\right)\right)\right\| \\
& \leq M\left[\|g(u)\|+\left\|\rho_{r}\right\|_{L^{1}}+\sum_{i=1}^{s}\left\|I_{i}\left(u\left(t_{i}\right)\right)\right\|\right] \\
& \leq r
\end{aligned}
$$

for each $t \in[0, b]$. It implies that $G W_{r} \subseteq W_{r}$.

Now, we show that $G W_{r}$ is equicontinuous on $J_{0}=\left[0, t_{1}\right], J_{i}=\left(t_{i}, t_{i+1}\right]$ and is also equicontinuous at $t=t_{i}^{+}, i=1, \ldots, s$. Indeed, we only need to prove that $G W_{r}$ is equicontinuous on $\left[t_{1}, t_{2}\right]$, as the cases for other subintervals are the same. For $u \in W_{r}, t_{1} \leq s<t \leq t_{2}$, we have, using the semigroup property,

$$
\begin{aligned}
\|T(t) g(u)-T(s) g(u)\| & =\|T(s)[T(t-s)-T(0)] g(u)\| \\
& \leq M\|[T(t-s)-T(0)] g(u)\| .
\end{aligned}
$$

Thus, $G_{1} W_{r}$ is equicontinuous on $\left[t_{1}, t_{2}\right]$ due to the compactness of $g$ and the strong continuity of $T(\cdot)$. The same idea can be used to prove the equicontinuity of $G_{3} W_{r}$ on $\left[t_{1}, t_{2}\right]$. That is, for $u \in W_{r}, t_{1} \leq s<t \leq t_{2}$, we have

$$
\left\|T\left(t-t_{1}\right) I_{1}\left(u\left(t_{1}\right)\right)-T\left(s-t_{1}\right) I_{1}\left(u\left(t_{1}\right)\right)\right\| \leq M\left\|[T(t-s)-T(0)] I_{1}\left(u\left(t_{1}\right)\right)\right\|,
$$


which implies the equicontinuity of $G_{3} W_{r}$ on $\left[t_{1}, t_{2}\right]$ due to the compactness of $I_{1}$ and the strong continuity of $T(\cdot)$. Moreover, from Lemma 2.9, we have that $G_{2} W_{r}$ is equicontinuous on $[0, b]$. Therefore, we have that the functions in $G W_{r}=\left(G_{1}+G_{2}+G_{3}\right) W_{r}$ are equicontinuous on each $\left[t_{i}, t_{i+1}\right], i=0,1, \ldots, s$.

Set $W=\overline{\operatorname{conv}} G\left(W_{r}\right)$, where conv means the closure of convex hull. It is easy to verify that $G$ maps $W$ into itself and $W$ is equicontiuous on each $\overline{J_{i}}=\left[t_{i}, t_{i+1}\right], i=0,1, \ldots, s$. Now, we show that $G: W \rightarrow W$ is a convex-power condensing operator. Take $x_{0} \in W$, we shall prove that there exists a positive integral $n_{0}$ such that

$$
\beta\left(G^{\left(n_{0}, x_{0}\right)}(B)\right)<\beta(B),
$$

for every nonprecompact bounded subset $B \subset W$.

From Lemma 2.2 and Lemma 2.8, noticing the compactness of $g$ and $I_{i}$, we have

$$
\begin{aligned}
\beta\left(\left(G^{\left(1, x_{0}\right)} B\right)(t)\right)= & \beta((G B)(t)) \\
\leq & \beta(T(t) g(B))+\beta\left(\int_{0}^{t} T(t-s) f(s, B(s)) \mathrm{d} s\right) \\
& +\beta\left(\sum_{0<t_{i}<t} T\left(t-t_{i}\right) I_{i}\left(B\left(t_{i}\right)\right)\right) \\
\leq & \int_{0}^{t} \beta(T(t-s) f(s, B(s))) \mathrm{d} s \\
\leq & M \int_{0}^{t} \beta(f(s, B(s))) \mathrm{d} s \\
\leq & M \int_{0}^{t} L \beta(B(s)) \mathrm{d} s \\
\leq & M L t \beta(B)
\end{aligned}
$$

for $t \in[0, b]$. Further,

$$
\begin{aligned}
\beta\left(\left(G^{\left(2, x_{0}\right)} B\right)(t)\right)= & \beta\left(\left(G \operatorname{conv}\left\{G^{\left(1, x_{0}\right)} B, x_{0}\right\}\right)(t)\right) \\
\leq & \beta\left(T(t) g\left(\overline{\operatorname{conv}}\left\{G^{\left(1, x_{0}\right)} B, x_{0}\right\}\right)\right) \\
& +\beta\left(\int_{0}^{t} T(t-s) f\left(s, \overline{\operatorname{conv}}\left\{G^{\left(1, x_{0}\right)} B(s), x_{0}(s)\right\}\right) \mathrm{d} s\right) \\
& +\beta\left(\sum_{0<t_{i}<t} T\left(t-t_{i}\right) I_{i}\left(\overline{\operatorname{conv}}\left\{G^{\left(1, x_{0}\right)} B\left(t_{i}\right), x_{0}\left(t_{i}\right)\right\}\right)\right) \\
\leq & \beta\left(\int_{0}^{t} T(t-s) f\left(s, \overline{\operatorname{conv}}\left\{G^{\left(1, x_{0}\right)} B(s), x_{0}(s)\right\}\right) \mathrm{d} s\right) \\
\leq & M \int_{0}^{t} \beta\left(f\left(s, \overline{\operatorname{conv}}\left\{G^{\left(1, x_{0}\right)} B(s), x_{0}(s)\right\}\right)\right) \mathrm{d} s \\
\leq & M \int_{0}^{t} L \beta\left(\overline{\operatorname{conv}}\left\{G^{\left(1, x_{0}\right)} B(s), x_{0}(s)\right\}\right) \mathrm{d} s \\
\leq & M L \int_{0}^{t} \beta\left(\left(G^{\left(1, x_{0}\right)} B\right)(s)\right) \mathrm{d} s
\end{aligned}
$$




$$
\begin{aligned}
& \leq M L \int_{0}^{t} M L s \beta(B) \mathrm{d} s \\
& \leq M^{2} L^{2} \frac{t^{2}}{2 !} \beta(B)
\end{aligned}
$$

for $t \in[0, b]$. We can continue this iterative procedure and get that

$$
\beta\left(\left(G^{\left(n, x_{0}\right)} B\right)(t)\right) \leq \frac{M^{n} L^{n} b^{n}}{n !} \beta(B)
$$

for $t \in[0, b]$. As $G^{\left(n, x_{0}\right)}(B)$ is equicontinuous on each $\left[t_{i}, t_{i+1}\right]$, by Lemma 2.7 , we have that

$$
\beta\left(G^{\left(n, x_{0}\right)} B\right)=\sup _{t \in[0, b]} \beta\left(\left(G^{\left(n, x_{0}\right)} B\right)(t)\right) \leq \frac{M^{n} L^{n} b^{n}}{n !} \beta(B) .
$$

By the fact that $\frac{M^{n} L^{n} b^{n}}{n !} \rightarrow 0$ as $n \rightarrow \infty$, we know that there exists a large enough positive integral $n_{0}$ such that

$$
\frac{M^{n_{0}} L^{n_{0}} b^{n_{0}}}{n_{0} !}<1
$$

which implies that $G: W \rightarrow W$ is a convex-power condensing operator. From Lemma 2.5, $G$ has at least one fixed point in $W$, which is just a mild solution of the nonlocal impulsive problem (1.1). This completes the proof of Theorem 3.1.

Remark 3.2 By using the method of the measure of noncompactness, we require $f$ to satisfy some proper conditions of MNC, but do not require the compactness of a semigroup $T(t)$. Note that if $f$ is compact or Lipschitz continuous, then the condition (Hf)(ii) is satisfied. And our work improves many previous results, where they need the compactness of $T(t)$ or $f$, or the Lipschitz continuity of $f$. In the proof, Lemma 2.7 plays an important role for the impulsive differential equations, which provides us with the way to calculate the measure of noncompactness in $P C([0, b] ; X)$. The use of noncompact measures in functional differential and integral equations can also be seen in [18-20,22].

Remark 3.3 When we apply Darbo-Sadovskii's fixed point theorem to get the fixed point of a map, a strong inequality is needed to guarantee its condensing property. By using the $\beta$-convex-power condensing operator developed by Sun et al. [29], we do not impose any restrictions on the coefficient $L$. This generalized condensing operator also can be seen in Liu et al. [30], where nonlinear Volterra integral equations are discussed.

In the following, by using Lemma 2.7 and Darbo-Sadovskii's fixed point theorem, we give the existence results of the problem (1.1) under Lipschitz conditions and mixed-type conditions, respectively.

We give the following hypotheses:

(Hg2) $g: P C([0, b] ; X) \rightarrow X$ is Lipschitz continuous with the Lipschitz constant $k$.

(HI2) $I_{i}: X \rightarrow X$ is Lipschitz continuous with the Lipschitz constant $k_{i}$; that is,

$$
\left\|I_{i}(x)-I_{i}(y)\right\| \leq k_{i}\|x-y\|
$$

for $x, y \in X, i=1, \ldots, s$. 
Theorem 3.4 Assume that the hypotheses (HA), (Hf), (Hg2), (HI2) are satisfied, then the nonlocal impulsive problem $(1.1)$ has at least one mild solution on $[0, b]$ provided that

$$
M\left(k+L b+\sum_{i=1}^{s} k_{i}\right)<1
$$

and (3.3) are satisfied.

Proof From the proof of Theorem 3.1, we have that the solution operator $G$ is continuous and maps $W_{r}$ into itself. It remains to show that $G$ is $\beta$-condensing in $W_{r}$.

By the conditions (Hg2) and (HI2), we get that $G_{1}+G_{3}: W_{r} \rightarrow P C([0, b] ; X)$ is Lipschitz continuous with the Lipschitz constant $M\left(k+\sum_{i=1}^{s} k_{i}\right)$. In fact, for $u, v \in W_{r}$, we have

$$
\begin{aligned}
& \left\|\left(G_{1}+G_{3}\right) u-\left(G_{1}+G_{3}\right) v\right\|_{P C} \\
& =\sup _{t \in[0, b]}\left[\|T(t)(g(u)-g(v))\|+\sum_{0<t_{i}<t}\left\|T\left(t-t_{i}\right)\left(I_{i}\left(u\left(t_{i}\right)\right)-I_{i}\left(v\left(t_{i}\right)\right)\right)\right\|\right] \\
& \leq M\left[\|g(u)-g(v)\|+\sum_{i=1}^{s}\left\|I_{i}\left(u\left(t_{i}\right)\right)-I_{i}\left(v\left(t_{i}\right)\right)\right\|\right] \\
& \leq M\left(k+\sum_{i=1}^{s} k_{i}\right)\|u-v\|_{P C} .
\end{aligned}
$$

Thus, from Lemma 2.2(7), we obtain that

$$
\beta\left(\left(G_{1}+G_{3}\right) W_{r}\right) \leq M\left(k+\sum_{i=1}^{s} k_{i}\right) \beta\left(W_{r}\right) .
$$

For the operator $\left(G_{2} u\right)(t)=\int_{0}^{t} T(t-s) f(s, u(s)) \mathrm{d} s$, from Lemma 2.6, Lemma 2.8 and Lemma 2.9, we have

$$
\begin{aligned}
\beta\left(G_{2} W_{r}\right) & =\sup _{t \in[0, b]} \beta\left(\left(G_{2} W_{r}\right)(t)\right) \\
& \leq \sup _{t \in[0, b]} \int_{0}^{t} \beta\left(T(t-s) f\left(s, W_{r}(s)\right)\right) \mathrm{d} s \\
& \leq \sup _{t \in[0, b]} M \int_{0}^{t} L \beta\left(W_{r}(s)\right) \mathrm{d} s \\
& \leq M L b \beta\left(W_{r}\right) .
\end{aligned}
$$

Combining (3.5) and (3.6), we have

$$
\begin{aligned}
\beta\left(G W_{r}\right) & \leq \beta\left(\left(G_{1}+G_{3}\right) W_{r}\right)+\beta\left(G_{2} W_{r}\right) \\
& \leq M\left(k+L b+\sum_{i=1}^{s} k_{i}\right) \beta\left(W_{r}\right) .
\end{aligned}
$$

From the condition (3.4), $M\left(k+L b+\sum_{i=1}^{s} k_{i}\right)<1$, the solution map $G$ is $\beta$-condensing in $W_{r}$. By Darbo-Sadovskii's fixed point theorem, $G$ has a fixed point in $W_{r}$, which is just 
a mild solution of the nonlocal impulsive problem (1.1). This completes the proof of Theorem 3.4.

Among the previous works on nonlocal impulsive differential equations, few are concerned with the mixed-type conditions. Here, by using Lemma 2.7, we can also deal with the mixed-type conditions in a similar way.

Theorem 3.5 Assume that the hypotheses (HA), (Hf), (Hg1), (HI2) are satisfied, then the nonlocal impulsive problem (1.1) has at least one mild solution on $[0, b]$ provided that

$$
M\left(L b+\sum_{i=1}^{s} k_{i}\right)<1
$$

and (3.3) are satisfied.

Proof We will also use Darbo-Sadovskii's fixed point theorem to obtain a fixed point of the solution operator $G$. From the proof of Theorem 3.1, we have that $G$ is continuous and maps $W_{r}$ into itself.

Subsequently, we show that $G$ is $\beta$-condensing in $W_{r}$. From the compactness of $g$ and the strong continuity of $T(\cdot)$, we get that $\left\{T(\cdot) g(u): u \in W_{r}\right\}$ is equicontinuous on $[0, b]$. Then by Lemma 2.6, we have that

$$
\beta\left(G_{1} W_{r}\right)=\sup _{t \in[0, b]} \beta\left(\left(G_{1} W_{r}\right)(t)\right)=\sup _{t \in[0, b]} \beta\left(T(t) g\left(W_{r}\right)\right)=0 .
$$

On the other hand, for $u, v \in W_{r}$, we have

$$
\begin{aligned}
\left\|G_{3} u-G_{3} v\right\|_{P C} & =\sup _{t \in[0, b]}\left\|\sum_{0<t_{i}<t} T\left(t-t_{i}\right)\left(I_{i}\left(u\left(t_{i}\right)\right)-I_{i}\left(v\left(t_{i}\right)\right)\right)\right\| \\
& \leq M \sum_{i=1}^{s}\left\|I_{i}\left(u\left(t_{i}\right)\right)-I_{i}\left(v\left(t_{i}\right)\right)\right\| \\
& \leq M \sum_{i=1}^{s} k_{i}\|u-v\|_{P C} .
\end{aligned}
$$

Then by Lemma 2.2(7), we obtain that

$$
\beta\left(G_{3} W_{r}\right) \leq M \sum_{i=1}^{s} k_{i} \beta\left(W_{r}\right)
$$

Combining (3.6), (3.8) and (3.9), we get that

$$
\beta\left(G W_{r}\right) \leq \beta\left(G_{1} W_{r}\right)+\beta\left(G_{2} W_{r}\right)+\beta\left(G_{3} W_{r}\right) \leq M\left(L b+\sum_{i=1}^{s} k_{i}\right) \beta\left(W_{r}\right) .
$$

From the condition (3.7), the map $G$ is $\beta$-condensing in $W_{r}$. So, $G$ has a fixed point in $W_{r}$ due to Darbo-Sadovskii's fixed point theorem, which is just a mild solution of the nonlocal impulsive problem (1.1). This completes the proof of Theorem 3.5. 
Theorem 3.6 Assume that the hypotheses (HA), (Hf), (Hg2), (HI1) are satisfied, then the nonlocal impulsive problem (1.1) has at least one mild solution on $[0, b]$ provided that

$$
M(k+L b)<1
$$

and (3.3) are satisfied.

Proof From the proof of Theorem 3.1, we have that the solution operator $G$ is continuous and maps $W_{r}$ into itself. In the following, we shall show that $G$ is $\beta$-condensing in $W_{r}$.

By the Lipschitz continuity of $g$, we have that for $u, v \in W_{r}$,

$$
\left\|G_{1} u-G_{1} v\right\|_{P C}=\sup _{t \in[0, b]}\|T(t)[g(u)-g(v)]\| \leq M k\|u-v\|_{P C}
$$

which implies that

$$
\beta\left(G_{1} W_{r}\right) \leq M k \beta\left(W_{r}\right)
$$

Similar to the discussion in Theorem 3.1, from the compactness of $I_{i}$ and the strong continuity $T(\cdot)$, we get that $G_{3} W_{r}$ is equicontinuous on each $\bar{J}_{i}=\left[t_{i}, t_{i+1}\right], i=0,1, \ldots, s$. Then by Lemma 2.7 , we have that

$$
\beta\left(G_{3} W_{r}\right)=\sup _{t \in[0, b]} \beta\left(\left(G_{3} W_{r}\right)(t)\right) \leq \sum_{i=1}^{s} \beta\left(T\left(t-t_{i}\right) I_{i}\left(W_{r}\left(t_{i}\right)\right)\right)=0 .
$$

Combining (3.6), (3.11) and (3.12), we have that

$$
\beta\left(G W_{r}\right) \leq \beta\left(G_{1} W_{r}\right)+\beta\left(G_{2} W_{r}\right)+\beta\left(G_{3} W_{r}\right) \leq M(k+L b) \beta\left(W_{r}\right) .
$$

From condition (3.10), the map $G$ is $\beta$-condensing in $W_{r}$. So, $G$ has a fixed point in $W_{r}$ due to Darbo-Sadovskii's fixed point theorem, which is just a mild solution of the nonlocal impulsive problem (1.1). This completes the proof of Theorem 3.6.

Remark 3.7 With the assumption of compactness on the associated semigroup, the existence of mild solutions to functional differential equations has been discussed in [6, 23-25]. By using the method of the measure of noncompactness, we deal with the four cases of impulsive differential equations in a unified way and get the existence results when the semigroup in not compact.

\section{An example}

In the application to partial differential equations, such as a class of parabolic equations, the semigroup corresponding to the differential equations is an analytic semigroup. We know that an analytic semigroup or a compact semigroup must be equicontinuous; see Pazy [31]. So, our results can be applied to these problems. If the operator $A=\theta$, the corresponding semigroup $T(t)=I$ is equicontinuous on $[0, b]$. 
We consider the following partial differential system (based on [23]) to illustrate our abstract results:

$$
\left\{\begin{array}{l}
\frac{\partial}{\partial t} \omega(t, x)=\frac{\partial^{2}}{\partial x^{2}} \omega(t, x)+F(t, \omega(t, x)), \quad 0 \leq t \leq b, 0 \leq x \leq \pi \\
\omega(t, 0)=\omega(t, \pi)=0, \\
\omega\left(t_{i}^{+}, x\right)-\omega\left(t_{i}^{-}, x\right)=I_{i}\left(\omega\left(t_{i}, x\right)\right), \quad i=1, \ldots, s \\
\omega(0, x)=g(\omega(t, x)) .
\end{array}\right.
$$

Take $X=L^{2}[0, \pi]$ and the operator $A: D(A) \subseteq X \rightarrow X$ defined by $A z=z^{\prime \prime}$, with

$$
D(A)=\left\{z \in X: z, z^{\prime} \text { are absolutely continuous, } z^{\prime \prime} \in X, z(0)=z(\pi)=0\right\} .
$$

From Pazy [31], we know that $A$ is the infinitesimal generator of an analytic semigroup $T(t), t \geq 0$. This implies that $A$ satisfies the condition (HA).

Let $0<t_{1}<t_{2}<\cdots<t_{s}<b, 0<s_{1}<s_{2}<\cdots<s_{q}<b, c_{j} \in R(j=0,1, \ldots, q), h(\cdot) \in$ $L^{1}([0, b] ; R), \alpha_{i}>0$ and $\rho_{i} \in C([0, \pi] \times[0, \pi], R)$ for $i=1, \ldots, s$. Now, we define that

(1) $f(t, u(\xi))=c_{0} \sin (u(\xi)), t \in[0, b], u \in X$

(2) $g(\omega(t, \xi))=\sum_{j=1}^{q} c_{j} \omega\left(s_{j}, \xi\right), \omega \in P C([0, b] ; X)$.

(3) $g(\omega(t, \xi))=\int_{0}^{b} h(s) \lg (1+|\omega(s, \xi)|) \mathrm{d} s, \omega \in P C([0, b] ; X)$.

(4) $I_{i}(u(\xi))=\frac{1}{\alpha_{i}|u(\xi)|+t_{i}}, u \in X, 1 \leq i \leq s$.

(5) $I_{i}(u(\xi))=\int_{0}^{\pi} \rho_{i}(\xi, y) \cos ^{2}(u(y)) \mathrm{d} y, u \in X, 1 \leq i \leq s$.

Then we obtain that

Case 1. Under the conditions (1) + (3) + (5), the assumptions in Theorem 3.1 are satisfied for large $r>0$. Therefore, the corresponding system (1.1) has at least a mild solution.

Case 2. Under the conditions (1) + (2) + (4), the assumptions in Theorem 3.4 are satisfied for large $r>0$. Therefore, the corresponding system (1.1) has at least a mild solution.

Case 3. Under the conditions (1) + (3) + (4), the assumptions in Theorem 3.5 are satisfied for large $r>0$. Therefore, the corresponding system (1.1) has at least a mild solution.

Case 4. Under the conditions (1) + (2) + (5), the assumptions in Theorem 3.6 are satisfied for large $r>0$. Therefore, the corresponding system (1.1) has at least a mild solution.

\section{Competing interests}

The authors declare that they have no competing interests.

\section{Authors' contributions}

All authors read and approved the final manuscript.

\section{Acknowledgements}

Research is partially supported by the National Natural Science Foundation of China (11271316), the Postgraduate Innovation Project of Jiangsu Province (No. CXZZ12-0890), the NSF of China (11101353), the first author is also supported by the Youth Teachers Foundation of Huaiyin Institute of Technology (2012).

Received: 3 June 2012 Accepted: 9 October 2012 Published: 24 October 2012

\section{References}

1. Lakshmikantham, V, Bainov, DD, Simeonov, PS: Theory of Impulsive Differential Equations. World Scientific, Singapore (1989)

2. Benchohra, M, Henderson, J, Ntouyas, SK: Impulsive Differential Equations and Inclusions. Hindawi Publishing, New York (2006)

3. Guo, M, Xue, X, Li, R: Controllability of impulsive evolution inclusions with nonlocal conditions. J. Optim. Theory Appl. $120,355-374(2004)$

4. Hernández, E, Rabelo, M, Henríquez, HR: Existence of solutions for impulsive partial neutral functional differential equations. J. Math. Anal. Appl. 331, 1135-1158 (2007) 
5. Ji, S, Wen, S: Nonlocal Cauchy problem for impulsive differential equations in Banach spaces. Int. J. Nonlinear Sci. 10(1), 88-95 (2010)

6. Ji, S, Li, G: Existence results for impulsive differential inclusions with nonlocal conditions. Comput. Math. Appl. 62 , 1908-1915 (2011)

7. Li, J, Nieto, JJ, Shen, J: Impulsive periodic boundary value problems of first-order differential equations. J. Math. Anal. Appl. 325, 226-236 (2007)

8. Benchohra, M, Henderson, J, Ntouyas, SK: An existence result for first-order impulsive functional differential equations in Banach spaces. Comput. Math. Appl. 42, 1303-1310 (2001)

9. Liu, JH: Nonlinear impulsive evolution equations. Dyn. Contin. Discrete Impuls. Syst. 6, 77-85 (1999)

10. Nieto, JJ, Rodriguez-Lopez, R: Periodic boundary value problem for non-Lipschitzian impulsive functional differential equations. J. Math. Anal. Appl. 318, 593-610 (2006)

11. Cardinali, T, Rubbioni, P: Impulsive semilinear differential inclusion: topological structure of the solution set and solutions on non-compact domains. Nonlinear Anal. 14, 73-84 (2008)

12. Abada, N, Benchohra, M, Hammouche, H: Existence and controllability results for nondensely defined impulsive semilinear functional differential inclusions. J. Differ. Equ. 246, 3834-3863 (2009)

13. Byszewski, L, Lakshmikantham, V: Theorem about the existence and uniqueness of solutions of a nonlocal Cauchy problem in a Banach space. Appl. Anal. 40, 11-19 (1990)

14. Byszewski, L: Theorems about the existence and uniqueness of solutions of a semilinear evolution nonlocal Cauchy problem. J. Math. Anal. Appl. 162, 494-505 (1991)

15. Fu, X, Ezzinbi, K: Existence of solutions for neutral functional differential evolution equations with nonlocal conditions. Nonlinear Anal. 54, 215-227 (2003)

16. Aizicovici, S, McKibben, M: Existence results for a class of abstract nonlocal Cauchy problems. Nonlinear Anal. 39, 649-668 (2000)

17. Xue, X: Nonlinear differential equations with nonlocal conditions in Banach spaces. Nonlinear Anal. 63, 575-586 (2005)

18. Xue, X: Nonlocal nonlinear differential equations with a measure of noncompactness in Banach spaces. Nonlinear Anal. 70, 2593-2601 (2009)

19. Banas, J, Zajac, T: A new approach to the theory of functional integral equations of fractional order. J. Math. Anal. Appl. 375, 375-387 (2011)

20. Cardinali, T, Rubbioni, $\mathrm{P}$ : On the existence of mild solutions of semilinear evolution differential inclusions. J. Math. Anal. Appl. 308, 620-635 (2005)

21. Dong, Q, Li, G: Existence of solutions for semilinear differential equations with nonlocal conditions in Banach spaces Electron. J. Qual. Theory Differ. Equ. 2009, 47 (2009)

22. Agarwal, RP, Benchohra, M, Seba, D: On the application of measure of noncompactness to the existence of solutions for fractional differential equations. Results Math. 55, 221-230 (2009)

23. Liang, J, Liu, JH, Xiao, TJ: Nonlocal impulsive problems for nonlinear differential equations in Banach spaces. Math. Comput. Model. 49, 798-804 (2009)

24. Fan, Z: Impulsive problems for semilinear differential equations with nonlocal conditions. Nonlinear Anal. 72, 1104-1109 (2010)

25. Fan, Z, Li, G: Existence results for semilinear differential equations with nonlocal and impulsive conditions. J. Funct. Anal. 258, 1709-1727 (2010)

26. Zhu, L, Dong, Q, Li, G: Impulsive differential equations with nonlocal conditions in general Banach spaces. Adv. Differ. Equ. 2012, 10 (2012)

27. Barbu, V: Nonlinear Semigroups and Differential Equations in Banach Spaces. Noordhoff, Leyden (1976)

28. Banas, J, Goebel, K: Measure of Noncompactness in Banach Spaces. Lect. Notes Pure Appl. Math., vol. 60. Dekker, New York (1980)

29. Sun, J, Zhang, X: The fixed point theorem of convex-power condensing operator and applications to abstract semilinear evolution equations. Acta Math. Sin. Chin. Ser. 48, 439-446 (2005)

30. Liu, LS, Guo, F, Wu, CX, Wu, YH: Existence theorems of global solutions for nonlinear Volterra type integral equations in Banach spaces. J. Math. Anal. Appl. 309, 638-649 (2005)

31. Pazy, A: Semigroups of Linear Operators and Applications to Partial Differential Equations. Springer, New York (1983)

doi:10.1186/1687-1847-2012-182

Cite this article as: Ji and Li: A unified approach to nonlocal impulsive differential equations with the measure of noncompactness. Advances in Difference Equations 2012 2012:182. 